\title{
Analytical perturbation theory and nucleon structure function in infrared region
}

\author{
L. Ghasemzadeh, ${ }^{1, \dagger}$ A. Mirjalili ${ }^{1,},{ }^{, *}$ and S. Atashbar Tehrani $\odot^{2, \$}$ \\ ${ }^{1}$ Physics Department, Yazd University, P.O.Box 89195-741, Yazd, Iran \\ ${ }^{2}$ School of Particles and Accelerators, Institute for Research in Fundamental Sciences (IPM), \\ P.O. Box 19395-5531, Tehran, Iran
}

(Received 23 August 2021; accepted 9 September 2021; published 12 October 2021)

\begin{abstract}
We employ the analytic QCD (anQCD) approach to analyze the unpolarized nucleon structure function in deep inelastic scattering processes at next-to-leading-order accuracy. Considering the unreliable results of the underlying perturbative QCD (pQCD) at energy scales near to QCD cut off parameter $Q^{2} \sim \Lambda^{2}$ and below, we modify the calculations at these scales using the anQCD approach and compare them with results from underlying $\mathrm{pQCD}$ and the available experimental data. The massive perturbation theory model is also used where an effective mass is attributed to gluons. Finally, we use the Jacobi polynomials formalism to transfer the calculations from Mellin moment space to Bjorken- $x$ space. To confirm the validity of the anQCD approach the Gottfried sum rule is also investigated. The achieved numerical results at low energy scales are compatible with what is expected and correspond to an admissible behavior of parton densities.
\end{abstract}

DOI: 10.1103/PhysRevD.104.074007

\section{INTRODUCTION}

An observable should be an analytic (holomorphic) function in the complex $Q^{2}$ plane where $Q^{2} \nless 0$. At high energy scales, i.e., $\left|Q^{2}\right| \gg 1$, we can have a good theoretical description and achieve reliable results that confirm experimental data using the underlying perturbative QCD (pQCD). However, at energy scales near the QCD cutoff parameter, i.e., $Q^{2} \sim \Lambda^{2}$ and below, the coupling constant of QCD starts to grow rapidly, and as a result we face Landau IR singularities. On the other hand, spacelike QCD observables such as the nucleon structure functions do not have such singularities. Accordingly, one cannot obtain any reliable results from underlying $\mathrm{pQCD}$, and thus we need an efficient approach that eliminates these singularities in order to achieve suitable results. There are various approaches to attain this goal, such as the Brodsky coupling constant obtained using the AdS/CFT correspondance [1], the dispersive approach of Dokshitzer [2,3], and finally analytic perturbation theory (APT) [4-9]. We use the last one to shift and even eliminate the mentioned singularities in calculations of physical quantities, such as the unpolarized

\footnotetext{
* Corresponding author. a.mirjalili@yazd.ac.ir

†leilaghasemzadeh@stu.yazd.ac.ir

*atashbar@ipm.ir

Published by the American Physical Society under the terms of the Creative Commons Attribution 4.0 International license. Further distribution of this work must maintain attribution to the author(s) and the published article's title, journal citation, and DOI. Funded by SCOAP .
}

nucleon structure function (NSF) and the Gottfried sum rule, and thus modify their theoretical predictions. We refer to Ref. [10] for recent related work. In this approach, the running QCD coupling constant $\left[a\left(Q^{2}\right)=\frac{\alpha_{s}\left(Q^{2}\right)}{\pi}\right]$ is transformed into an analytic function of $Q^{2}$ (analytic for $Q^{2} \nless 0$ ), called the analytic QCD coupling constant $\left[A_{1}\left(Q^{2}\right)\right]$, and it does not have any Landau singularities. We are able to obtain the results for the mentioned quantities which do not contain any singularities at the low energies using the analytic coupling constant.

Approaches that eliminate the Landau singularities include fractional analytic perturbation theory (FAPT) [11-14]; $2 \delta \mathrm{anQCD}[14,15]$ and $3 \delta \mathrm{anQCD}[16]$ which are based on parametrizing the spectral function at low energies by two or three Dirac delta functions, respectively; and finally massive perturbation theory (MPT) [14,17] which is based on removing the Landau singularities by shifting them into the timelike region. The last method considers an effective mass for the gluon. Since we are working on the nucleon structure function, which contains the singlet and gluon sectors, we decide to apply it so that we can achieve better computational results.

The organization of this paper is as follows. In the next section we give a brief description of the essential concepts of APT. In Sec. III the evolution of parton densities and the nucleon structure function using the Jacobi transformation is discussed. Section IV is devoted to describing the structure function in the MPT model. Based on this model, the Gottfried sum rule is considered in Sec. V. Finally, a summary and conclusion is presented in Sec. VI. 


\section{BASIC CONCEPTS IN ANALYTIC PERTURBATION THEORY}

As we mentioned, the underlying $\mathrm{pQCD}$ coupling suffers from unphysical Landau singularities at $Q^{2} \sim \Lambda^{2}$. Therefore, we cannot apply it in the low-momentum regime, and this is a motivation to use other approaches, especially analytic QCD (anQCD), to achieve fairly accurate results for physical quantities. In this approach we have analytic couplings $A_{\nu}$ which are free from the aforementioned problems. In the following we will describe the main elements of APT. The application of Cauchy's theorem to the running coupling $a\left(Q^{2}\right)$, where $a\left(Q^{2}\right)=\alpha_{s}\left(Q^{2}\right) / \pi$, gives us the following spectral relation in general anQCD $[14,18]$ :

$$
A_{1}\left(Q^{2}\right)=\frac{1}{\pi} \int_{\sigma=M_{t h}^{2}}^{\infty} \frac{d \sigma \rho_{1}(\sigma)}{\left(\sigma+Q^{2}\right)},
$$

where

$$
\rho_{1}(\sigma) \equiv \operatorname{Im} A_{1}(-\sigma-i \varepsilon)
$$

Different approaches to consider the discontinuity function $\rho_{1}(\sigma)$ and the coupling function $A_{1}\left(Q^{2}\right)$ will lead to the various anQCD models. As we pointed out before, $A_{1}\left(Q^{2}\right)$ is the anQCD analog of the underlying pQCD coupling $a\left(Q^{2}\right)=\alpha_{s}\left(Q^{2}\right) / \pi$, i.e., at $\sigma \gg \Lambda$ we have $\equiv \operatorname{Im} a(-\sigma-i \varepsilon)=\equiv \operatorname{Im} A_{1}(-\sigma-i \varepsilon)$. Let us denote by $A_{\nu}$ the anQCD analog of the pQCD power $a^{\nu}$ (where $\nu$ is not necessarily an integer). An important point we should note is that there is no standard algebra for $A_{\nu}$, i.e., $A_{\nu} A_{\mu} \not \equiv$ $A_{\nu+\mu}$ or $A_{\nu+\mu} \not \equiv\left(A_{\nu}\right)^{\mu}$. For the construction of $A_{\nu}$ in a general anQCD, we follow Ref. [19].

Correct analogs $A_{n}\left(Q^{2}\right)$ of the powers $a^{n}\left(Q^{2}\right)$ will be achieved, using the logarithmic derivatives of $A_{1}$ [14]:

$$
\tilde{\mathcal{A}}_{n+1} \equiv \frac{(-1)^{n}}{\beta_{0}^{n} n !}\left(\frac{\partial}{\partial \ln Q^{2}}\right)^{n} A_{1}\left(Q^{2}\right)
$$

It is obvious that with $n=0$ we get $\tilde{\mathcal{A}}_{1} \equiv A_{1}$. Here $\beta_{0}=$ $\frac{1}{4}\left(11-\frac{2}{3} N_{f}\right)$ is the first coefficient of the QCD $\beta$ function which is scheme independent where this function is governed by the renormalization group equation for the QCD running coupling constant. Substituting $A_{1}$ in Eq. (1) into Eq. (3) will lead to

$$
\tilde{\mathcal{A}}_{n+1}\left(Q^{2}\right)=\frac{1}{\pi} \frac{(-1)}{\beta_{0}^{n} \Gamma(n+1)} \int_{0}^{\infty} \frac{d \sigma}{\sigma} \rho_{1}(\sigma) L i_{-n}\left(\frac{-\sigma}{Q^{2}}\right) .
$$

In this equation $n$ is an integer number and it can be extended to noninteger index $\nu$ as follows [19]:

$$
\tilde{\mathcal{A}}_{\nu+1}\left(Q^{2}\right)=\frac{1}{\pi} \frac{(-1)}{\beta_{0}^{\nu} \Gamma(\nu+1)} \int_{0}^{\infty} \frac{d \sigma}{\sigma} \rho_{1}(\sigma) L i_{-\nu}\left(\frac{-\sigma}{Q^{2}}\right) .
$$

Here $L i_{-\nu}\left(\frac{-\sigma}{Q^{2}}\right)$ is the polylogarithm function. It should be noted that the integral in Eq. (5) is converging at low $\sigma$ for $\nu>-1$ where the polyloghartitm function is approximated by $L i_{-\nu}(-z) \sim \ln ^{-\nu} z$. The analytic analogs $A_{\nu}\left(Q^{2}\right)$ can be constructed as linear combinations of $\tilde{\mathcal{A}}_{\nu+m}$ 's:

$$
A_{\nu}\left(Q^{2}\right)=\tilde{\mathcal{A}}_{\nu}+\sum_{m \geq 1} \tilde{k}_{m}(\nu) \tilde{\mathcal{A}}_{\nu+m} .
$$

The coefficients $\tilde{k}_{m}(\nu)$ in Eq. (6) have been determined in Ref. [19]. Using the analytic coupling constant, we can do the required calculations for quantities that contain noninteger power expansions of the coupling constant.

The specific anQCD model that is used in this paper, called massive perturbation theory. In this model an effective mass is attributed to gluon to achieve us to a holomorphic coupling. Therefore one can write $[14,17]$

$$
A_{1}^{\mathrm{MPT}}\left(Q^{2}, N_{f}\right)=a\left(Q^{2}+m_{\mathrm{gl}}^{2}, N_{f}\right) .
$$

The mass scale $m_{\mathrm{gl}} \sim 0.5-1 \mathrm{GeV}$ refers to the gluon mass, where $m_{\mathrm{gl}}^{2}=0.7 \mathrm{GeV}^{2}$ is considered here. Since $m_{\mathrm{gl}}^{2}>\Lambda^{2}$, we get a coupling that is analytic even to scales less than $\Lambda$. At high energy $A_{1}^{\mathrm{MPT}}\left(Q^{2}\right)$ tends to the pQCD coupling $a\left(Q^{2}\right)$. Considering Ref. [14] the difference of coupling constant in MPT model with respect to the underlying $\mathrm{pQCD}$ coupling is given by [14]

$$
A_{1}^{\mathrm{MPT}}\left(Q^{2}, N_{f}\right)-a\left(Q^{2}, N_{f}\right) \sim \frac{m_{\mathrm{gl}}^{2}}{Q^{2} \ln ^{2}\left(\frac{Q^{2}}{\Lambda^{2}}\right)} .
$$

In the following sections we will observe the benefits of the MPT model compared with the pQCD approach. As an adjunct to this issue, in Fig. 1 we plot the running coupling constant in two MPT and FAPT models and compare them with the underlying pQCD coupling.

\section{JACOBI TRANSFORMATION AND PARTON DENSITY EVOLUTIONS}

To extract the unpolarized NSF in terms of the energy scale $Q^{2}$ we need to evolve the singlet and nonsinglet sectors of the SF with respect to energy scale. Here we start by singlet densities where splitting functions are governing their evolution. The singlet quark distribution of a hadron is defined by

$$
\Sigma\left(x, Q^{2}\right)=\sum_{i=1}^{N_{f}}\left[q_{i}\left(x, Q^{2}\right)+\overline{q_{i}}\left(x, Q^{2}\right)\right] .
$$




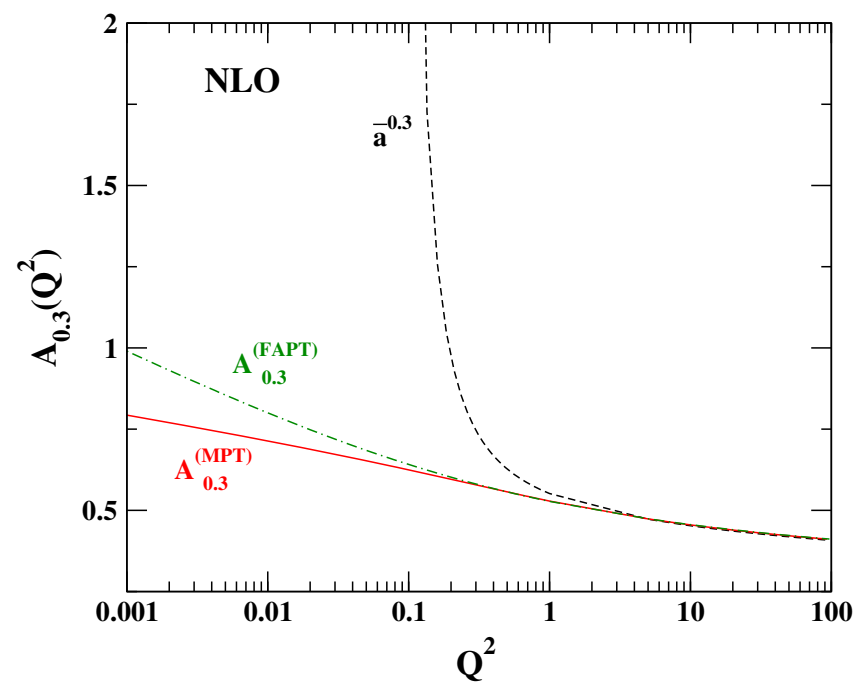

FIG. 1. Coupling constants using two MPT and FAPT models with fraction index $\nu=0.3$. The moderate behavior of the running coupling constant in these models at low energy scales in comparison to underlying $\mathrm{pQCD}$ is obvious.

Here $q_{i}\left(x, Q^{2}\right)$ and $\overline{q_{i}}\left(x, Q^{2}\right)$ represent the respective number densities of quarks and antiquarks as a function of the carried momentum fraction $x$. The subscript $i$ indicates the flavor of the (anti)quark and $n_{f}$ stands for the number of effectively massless flavors. Suppressing the fractional dependencies, the coupled evolution equations for the singlet patron and gluon distributions read

$$
\frac{d}{d \ln Q^{2}}\left(\begin{array}{c}
\Sigma \\
g
\end{array}\right)=\left(\begin{array}{ll}
P_{q q} & P_{q g} \\
P_{g q} & P_{g g}
\end{array}\right) \otimes\left(\begin{array}{c}
\Sigma_{0} \\
g_{0}
\end{array}\right),
$$

where $\otimes$ stands for a convolution integral in the momentum variable,

$$
[a \otimes b](x) \equiv \int_{x}^{1} \frac{d y}{y} a(y) b\left(\frac{x}{y}\right)
$$

The corresponding gluon distribution, $g\left(x, Q^{2}\right)$, is denoted here by $g$.

The quark-quark splitting function $P_{q q}$ [20] in Eq. (10) can be expressed as [21]

$$
P_{q q}=P_{n s}^{+}+N_{f}\left(P_{\bar{q} q}^{s}+P_{q q}^{s}\right) \equiv P_{n s}^{+}+P_{p s} .
$$

Here $P_{n s}^{+}$is the nonsinglet splitting function. The quantities $P_{q q}^{s}$ and $P_{\bar{q} q}^{s}$ are the flavor-independent sea contributions to the quark-quark and quark-antiquark splitting functions, respectively. The gluon-quark entries in Eq. (10) are given by

$$
P_{q g}=N_{f} P_{q_{i} g}, \quad P_{g q}=P_{g q_{i}} .
$$

In terms of the flavor-independent splitting functions, one can write $P_{q_{i} g}=P_{\bar{q} g}$ and $P_{g q_{i}}=P_{g \bar{q}}$.

The required calculations can now be continued in Mellin- $N$ space, using the Mellin transformation:

$$
a(N)=\int_{0}^{1} d x x^{N-1} a(x)
$$

Then, by transforming all needed quantities to Mellin (moment) space, the solution of Eq. (10) at next-toleading-order (NLO) accuracy is given by

$$
\begin{aligned}
\left(\begin{array}{c}
\Sigma \\
g
\end{array}\right)= & \left\{( \frac { a _ { s } } { a _ { 0 } } ) ^ { - r _ { - } } \left[\boldsymbol{e}_{-}+\left(a_{0}-a_{s}\right) \boldsymbol{e}_{-} \boldsymbol{R}_{1} \boldsymbol{e}_{-}\right.\right. \\
& \left.-\left(a_{0}-a_{s}\left(\frac{a_{s}}{a_{0}}\right)^{r_{-}-r_{+}}\right) \frac{\boldsymbol{e}_{-} \boldsymbol{R}_{1} \boldsymbol{e}_{+}}{r_{+}-r_{-}-1}\right] \\
& +(+\leftrightarrow-)\}\left(\begin{array}{c}
\Sigma_{0} \\
g_{0}
\end{array}\right) .
\end{aligned}
$$

Here we explicitly define $a_{s}=\alpha_{s} /(4 \pi)=a / 4$. In the last line the following recursive abbreviations have been used [21]:

$$
\begin{aligned}
\boldsymbol{R}_{0} & \equiv \frac{1}{\beta_{0}} \boldsymbol{P}^{(0)}, \\
\boldsymbol{R}_{k} & \equiv \frac{1}{\beta_{0}} \boldsymbol{P}^{(k)}-\sum_{i=1}^{k} b_{i} \boldsymbol{R}_{k-i},
\end{aligned}
$$

with $b_{k} \equiv \beta_{k} / \beta_{0}$. Furthermore, for the $r_{ \pm}$one can write

$$
r_{ \pm}=\frac{1}{2 \beta_{0}}\left[P_{q q}^{0}+P_{g g}^{0} \pm \sqrt{\left(P_{q q}^{0}-P_{g g}^{0}\right)^{2}-4 P_{q g}^{0} P_{g q}^{0}}\right]
$$

where the following relation for $\boldsymbol{e}_{ \pm}$is defined:

$$
\boldsymbol{e}_{ \pm}=\frac{1}{r_{ \pm}-r_{\mp}}\left[\boldsymbol{R}_{0}-r_{\mp} \boldsymbol{I}\right]
$$

where $I$ represents a unique $2 \times 2$ matrix.

In the nonsinglet case, in order to decouple the combination we need to use the general structure of the (anti) quark-(anti)quark splitting functions as follows [21]

$$
\begin{aligned}
& P_{q_{i} q_{k}}=P_{\bar{q}_{i} \bar{q}_{k}}=\delta_{i k} P_{q q}^{V}+P_{q q}^{s}, \\
& P_{q_{i} \bar{q}_{k}}=P_{\bar{q}_{i} q_{k}}=\delta_{i k} P_{q \bar{q}}^{V}+P_{q \bar{q}}^{s} .
\end{aligned}
$$

The flavor asymmetries $q_{n s}^{ \pm}$and the total valence distribution $q_{n s}^{V}$ and their corresponding splitting functions are given by [21] 


$$
\begin{aligned}
q_{n s, i k}^{ \pm} & =q_{i} \pm \bar{q}_{i}-\left(q_{k} \pm \bar{q}_{k}\right), \\
q_{n s}^{V} & =\sum_{r=1}^{n_{f}}\left(q_{r}-\bar{q}_{r}\right), \\
P_{n s}^{ \pm} & =P_{q q}^{V} \pm P_{q \bar{q}}^{V}, \\
P_{n s}^{V} & =P_{q q}^{V}-P_{q \bar{q}}^{V}+n_{f}\left(P_{q q}^{s}-P_{q \bar{q}}^{s}\right) \equiv P_{n s}^{-}+P_{n s}^{s} .
\end{aligned}
$$

For nonsinglet quark distribution evolution a similar process exists as in the singlet case, but with the obvious simplification that no spurious complexity occurs. Consequently, the nonsinglet evolution can be written as follows [21]:

$q^{ \pm, V}\left(a_{s}\right)=\exp \left[R_{1}^{ \pm, V}\left(a_{0}-a_{s}\right)\right]\left(\frac{a_{s}}{a_{0}}\right)^{-R_{0}^{ \pm, V}} q^{ \pm, V}\left(a_{0}\right)$,

where $R_{0}^{ \pm, V}=\frac{1}{\beta_{0}} P^{(0) \pm, V}$ and $R_{1}^{ \pm, V}=\frac{1}{\beta_{0}} P^{(1) \pm, V}-\beta_{1} R_{0}^{ \pm, V}$ are defined based on nonsinglet splitting functions where $\beta_{0}$ and $\beta_{1}$ are the first two universal coefficients of the QCD $\beta$ function. Accordingly, Eq. (21) at NLO accuracy can be written as

$q_{\mathrm{NLO}}^{ \pm, V}\left(a_{s}\right)=\left[1+R_{1}^{ \pm, V}\left(a_{0}-a_{s}\right)\right]\left(\frac{a_{s}}{a_{0}}\right)^{-R_{0}^{ \pm, V}} q^{ \pm, V}\left(a_{0}\right)$.

Finally, using Eqs. (15) and (22) we can obtain the nucleon structure function at the NLO accuracy in Mellin$N$ (moment) space as follows:

$$
\begin{aligned}
F_{2}\left(N, Q^{2}\right)= & {\left[C_{2 q}^{(0)}(N)+a_{s}\left(Q^{2}\right) C_{2 q}^{(1)}(N)\right] \sum_{i=u, d, s} e_{i}^{2} q_{i}\left(N, Q^{2}\right) } \\
& +a_{s}\left(Q^{2}\right) C_{2 g}^{(1)}(N) \frac{1}{f} \sum_{i=u, d, s} e_{i}^{2} g\left(N, Q^{2}\right) .
\end{aligned}
$$

Here $C^{k}(N)$ are Wilson coefficient functions which have been calculated in Ref. [22]. As mentioned before, using the Jacobi transformation is an adequate method to convert the calculated results from moment $N$ space to Bjorken- $x$ space. Details of this method have been described in Ref. [23,24]. According to this method, we can define the NSF based on the following relation:

$$
F_{2}\left(x, Q^{2}\right)=x^{\beta}(1-x)^{\alpha} \sum_{x=0}^{\infty} a_{n}^{\alpha, \beta}\left(Q^{2}\right) \Theta_{n}^{(\alpha, \beta)}(x) .
$$

Here $a_{n}^{\alpha, \beta}\left(Q^{2}\right)$ is an expansion coefficient and $\Theta_{n}^{(\alpha, \beta)}(x)$ denotes the Jacobi polynomials, and they are related to each other by

$$
a_{n}^{\alpha, \beta}\left(Q^{2}\right)=\int_{0}^{1} F_{2}\left(x, Q^{2}\right) \Theta_{n}^{(\alpha, \beta)}(x) d x .
$$

By substituting $\Theta_{n}^{(\alpha, \beta)}(x)=\sum_{k=0}^{n} C_{k, n}^{(\alpha, \beta)} x^{k}$ into Eq. (25), we get

$$
a_{n}^{\alpha, \beta}\left(Q^{2}\right)=\int_{0}^{1} F_{2}\left(x, Q^{2}\right) \sum_{j=0}^{n} C_{j, n}^{(\alpha, \beta)} x^{j} d x .
$$

Putting Eq. (26) into Eq. (24) and using the SF in moment $N$ space as $M_{F_{2}}\left(N, Q^{2}\right)=\int_{0}^{1} x^{j-2} F_{2}\left(x, Q^{2}\right) d x$, we can obtain SF in Bjorken- $x$ space as follows [25]:

$$
\begin{aligned}
F_{2}\left(x, Q^{2}\right)= & x^{\beta}(1-x)^{\alpha} \sum_{n=0}^{N_{\max }} \Theta_{n}^{(\alpha, \beta)}(x) \\
& \times \sum_{j=0}^{n} C_{j, n}^{(\alpha, \beta)} M_{F_{2}}\left(j+2, Q^{2}\right) .
\end{aligned}
$$

\section{UNPOLARIZED NUCLEON STRUCTURE FUNCTION AND THE MPT MODEL}

To do the required computations to extract the nucleon structure function we first need the parton distribution functions at the initial energy scale $Q_{0}$ as the inputs. For this purpose, the following parametrized functions are suggested [26]:

$$
\begin{aligned}
x u_{v}\left(x, Q_{0}^{2}\right) & =N_{u} x^{a_{u}}(1-x)^{b_{u}}\left(1+A_{u} \sqrt{x}+B_{u} x+C_{u} x^{2}\right), \\
x d_{v}\left(x, Q_{0}^{2}\right) & =N_{d} x^{a_{d}}(1-x)^{b_{d}}\left(1+A_{d} \sqrt{x}+B_{d} x+C_{d} x^{2}\right), \\
x g\left(x, Q_{0}^{2}\right) & =N_{g} x^{a_{g}}(1-x)^{b_{g}}\left(1+B_{g} x^{\alpha_{g}}(1-x)^{\beta_{g}}\right), \\
x \Sigma\left(x, Q_{0}^{2}\right) & =N_{\Sigma} x^{a_{\Sigma}}(1-x)^{b_{\Sigma}}\left(1+A_{\Sigma} \sqrt{x}+B_{\Sigma} x\right), \\
x \Delta\left(x, Q_{0}^{2}\right) & =N_{\Delta} x^{a_{\Delta}}(1-x)^{b_{\Delta}}\left(1+A_{\Delta} \sqrt{x}+B_{\Delta} x\right), \\
x s\left(x, Q_{0}^{2}\right) & =N_{s} x^{a_{s}}(1-x)^{b_{s}}\left(1+A_{s} \sqrt{x}+B_{s} x\right) .
\end{aligned}
$$

In Eq. (28) these definitions are used: $u_{v}=u-\bar{u}$, $d_{v}=d-\bar{d}, \quad \Sigma=\bar{u}+\bar{d}$, and $\Delta=\bar{d}-\bar{u}$. All unknown parameters including the normalization factors are obtained via the fitting over the related data [26]. The results are listed in Table I.

TABLE I. Numerical values of the free parameters in Eq. (28) at $Q_{0}^{2}=2 \mathrm{GeV}^{2}$ at NLO accuracy [26].

\begin{tabular}{lccccc}
\hline \hline \multicolumn{5}{c}{ NLO } \\
\hline$N_{u}$ & 1.63 & $N_{d}$ & 7.4 & $N_{g}$ & 2.95 \\
$a_{u}$ & 0.55 & $a_{d}$ & 0.92 & $a_{g}$ & 0.047 \\
$b_{u}$ & 3.61 & $b_{d}$ & 4.6 & $b_{g}$ & 6.1 \\
$A_{u}$ & 0.8 & $A_{d}$ & -2.8 & $B_{g}$ & 0 \\
$B_{u}$ & 4.7 & $B_{d}$ & 4.5 & $\alpha_{g}$ & 0 \\
$C_{u}$ & -0.1 & $C_{d}$ & -2 & $\beta_{g}$ & 0 \\
$N_{\Sigma}$ & 0.164 & $N_{\Delta}$ & 57 & $N_{s}$ & 0.03 \\
$a_{\Sigma}$ & -0.19 & $a_{\Delta}$ & 2.29 & $a_{s}$ & -0.28 \\
$b_{\Sigma}$ & 8.42 & $b_{\Delta}$ & 18.6 & $b_{s}$ & 8.42 \\
$A_{\Sigma}$ & 1.9 & $A_{\Delta}$ & 1 & $A_{s}$ & 1.9 \\
$B_{\Sigma}$ & 10 & $B_{\Delta}$ & 0 & $B_{s}$ & 10 \\
\hline \hline
\end{tabular}


The computations in this paper are done with Mathematica using the anQCD.m package [14], and we calculate the analytic coupling constant corresponding to the underlying $\mathrm{pQCD}$ coupling, realizing the powers given in Eq. (23). The relevant Mathematica command for the MPT coupling constant is $\operatorname{AMPTNl}\left[N_{f}, \nu, Q^{2}, m_{\mathrm{gl}}^{2}, \Lambda^{2}\right]$, which returns the $N$-loop $(N=1,2,3,4)$ analytic MPT coupling $A_{\nu}^{(\mathrm{MPT}, N)}\left(Q^{2}, m_{\mathrm{gl}}^{2}, N_{f}\right)$, including the fractional index $\nu$ at a fixed number of active quark flavors $N_{f}$, with $Q^{2}$ in the Euclidean domain $\left(Q^{2}>0\right)$. During our calculations at the NLO approximation, the $N$-loop is fixed at two (i.e., we use two-loop MPT). The other commands for analytic coupling constant in various anQCD models, have been described in Ref. [14]. The interested reader is also encouraged to read Ref. [19]. For simplicity, we use the notation $a_{s}^{\nu} \equiv \frac{a^{\nu}}{4^{\nu}}$ and subsequently $A_{s, \nu} \equiv \frac{A_{\nu}}{4^{\nu}}$, so the mentioned command becomes $\frac{A M P T 2 l\left[3, \nu, Q^{2}, m_{\mathrm{gl}}^{2}, \Lambda^{2}\right]}{4^{\nu}}$. Here $N_{f}=3$, $m_{\mathrm{gl}}^{2}=0.7 \mathrm{GeV}^{2}$, and $\Lambda^{2}=0.35 \mathrm{GeV}^{2}$.

Extracting the nucleon structure function, at first view, might be done by employing the MPT model on singlet and nonsinglet evolution equations, given by Eqs. (15) and (22) and then applying the model on Eq. (23) to consider the contribution of Wilson coefficients. This is not admissible since Wilson coefficients and parton densities are not directly observable and it is the nucleon structure function that should be analyzed, and not the different factors in Eq. (23). Based on this way, we should employ the MPT model entirely on Eq. (23) and not on its factors separately. Hence when Eq. (23) is written in anQCD approach, part of the exponent number of analytical coupling constant is arising from the evolved parton densities. The rest is related to exponent of coupling constant that is put behind Wilson factors. This procedure corresponds completely to the specific algebraic property of analytical couplings, i.e. $A_{\nu} A_{\mu} \neq A_{\nu+\mu}$ or $A_{\nu+\mu} \neq\left(A_{\nu}\right)^{\mu}$ which we discussed about it in Sec. II.

In fact, what we finally need to calculate can be given summarily by

$$
\begin{aligned}
& F_{2}\left(N, A_{\nu+1}\left(Q^{2}\right)\right) \\
& =\left[C_{2 q}^{(0)}(N)+A_{1}\left(Q^{2}\right) C_{2 q}^{(1)}(N)\right] \sum_{i=u, d, s} e_{i}^{2} q_{i}\left(N, A_{\nu}\left(Q^{2}\right)\right) \\
& \quad+A_{1}\left(Q^{2}\right) C_{2 g}^{(1)}(N) \times \frac{1}{f} \sum_{i=u, d, s} e_{i}^{2} g\left(N, A_{\nu}\left(Q^{2}\right)\right),
\end{aligned}
$$

where we replace $A_{1} A_{\nu} \mapsto A_{\nu+1}\left(\neq A_{1} A_{\nu}\right)$.

Considering the numerical values for the required parameters in the analytical coupling, the Mathematica command used for the $A_{\nu}\left(Q^{2}\right)$ coupling would be AMPT2l[3, $\left., Q^{2}, 0.7,0.35\right]$, where the $\nu$ index is determined via the evolution processes for singlet and gluon densities and the nonsinglet density. In practical calculations, this index takes the following forms: $\nu=-R_{0}, 1-R_{0}, 2-R_{0},-r_{-}, 1-r_{-}, 2-r_{-}, r_{+}, 1-r_{+}, 2-r_{+}$.

Using available data at different energy scales makes it possible to present the $Q$ dependence of the Jacobi parameters $\alpha$ and $\beta$ in Eq. (24) as it follows:

$$
\begin{aligned}
& \alpha=-465.737+553.088 \exp \left(Q^{2}\right)+\frac{336.376}{\log \left(Q^{2}\right)}, \\
& \beta=11.158+\frac{7.670 Q^{2}}{\log \left(Q^{2}\right)}+14.308 \sqrt{Q^{2}} \log \left(Q^{2}\right) .
\end{aligned}
$$

In Fig. 2 we show the $F_{2}^{p}\left(x, Q^{2}\right)$ structure function versus the Bjorken- $x$ variable at the different energy scales $Q^{2}=0.15,0.21,0.27$, and $0.313 \mathrm{GeV}^{2}$ using the MPT model and compare it with E665 experimental data [27]. To indicate the adequate applicability of the MPT model at low energy scales, we also add to this figure the results of underlying $\mathrm{pQCD}$ for the $F_{2}^{p}\left(x, Q^{2}\right)$ structure function. To achieve more precise results, the underlying $\mathrm{pQCD}$ computations are done in the two-loop approximation of the coupling constant [28-31].

Although there are not so much experimental data in above mentioned energy scales but as it is shown in Fig. 2, an appropriate agreement is standing between anQCD results and the available experimental data.
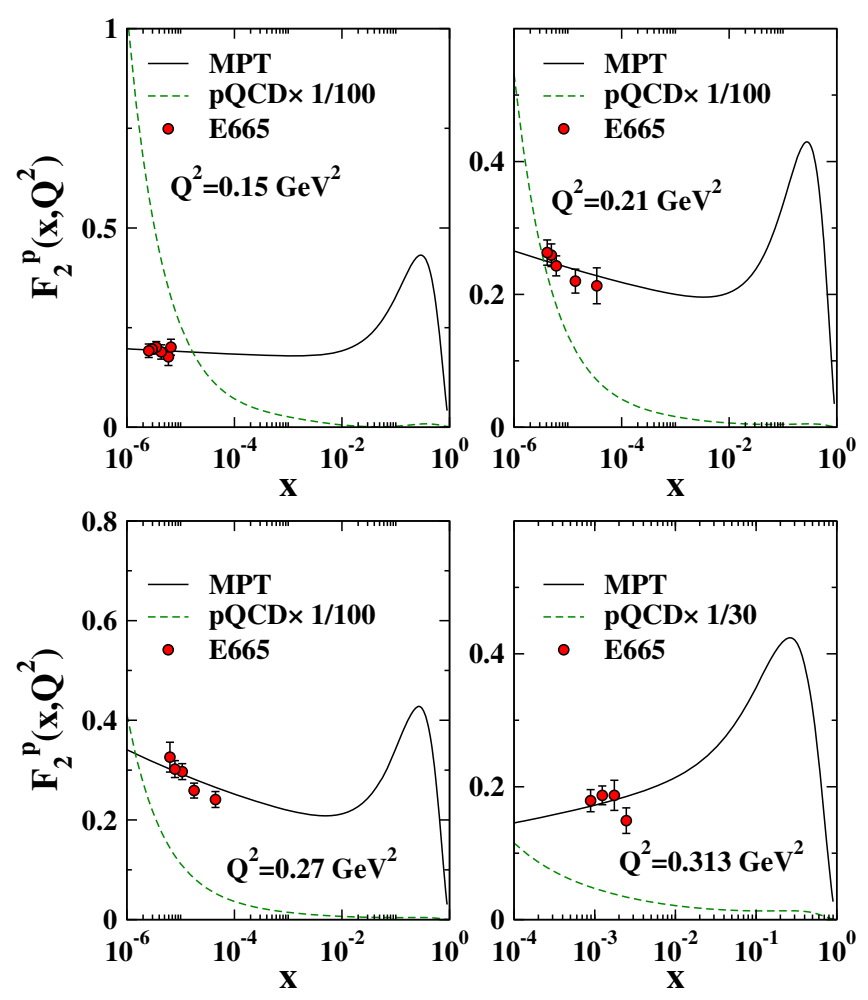

FIG. 2. Unpolarized nucleon structure function $F_{2}^{p}\left(x, Q^{2}\right)$ as a function of the Bjorken- $x$ variable, using the MPT model (solid line) and underlying pQCD (dashed line), and comparison with the available experimental data [27]. 


\section{GOTTFRIED SUM RULE IN THE anQCD APPROACH}

Since establishing the quark-parton model, the sum rules for nucleon structure functions play important roles to develop this model. One important sum rule is called the Gottfried sum rule (GSR). Considering the isospin symmetry for parton densities in the proton and neutron, the numerical value for the GSR would be different from the measured value by NMC group [32] where they take into account the electromagnetic structure function of the nucleon through the deep inelastic scattering of muons from protons and deuterons. Here we consider the GSR numerical values at low energy scales. Therefore we can use the MPT model to calculate the GSR at these energy scales. As we mentioned above, this sum rule provides a determination of the light flavor asymmetry of the nucleon sea and is given by [33]

$$
\begin{aligned}
S_{G} & \equiv \int_{0}^{1} \frac{d x}{x}\left[F_{2}^{p}\left(x, Q^{2}\right)-F_{2}^{n}\left(x, Q^{2}\right)\right] \\
& =\int_{0}^{1} \frac{1}{3}(u(x)-d(x)+\bar{u}(x)-\bar{d}(x)) d x \\
& =\frac{1}{3}-\frac{2}{3} \int_{0}^{1}(\bar{d}(x)-\bar{u}(x)) d x .
\end{aligned}
$$

$S_{G}$ deviates from the expectation of the simple quark model. In other words, if the nucleon sea were flavor symmetric, i.e., $\bar{u}(x)=\bar{d}(x)$, the GSR would be $S_{G}=\frac{1}{3}$, but this is in contrast with data from the NMC Collaboration on lepton-nucleon deep inelastic scattering $[32,34,35]$. Accordingly, the following numerical value for $S_{G}$ has been reported $[33,36]$ :

$$
S_{G}\left(Q^{2}=4 \mathrm{GeV}^{2}\right)=0.235 \pm 0.026 .
$$

This discrepancy can be associated with the existence of perturbative effects in the nucleon sea, which generate light-quark flavor asymmetry $\bar{u}\left(x, Q^{2}\right)<\bar{d}\left(x, Q^{2}\right)$ over a significant range of the Bjorken- $x$ variable [33]. For numerical values of the GSR at some specific energies, we refer to Ref. [36].

We apply the MPT model to calculate the GSR at energy scales lower than the QCD cutoff $\Lambda$, which is about $0.35 \mathrm{GeV}^{2}$. The obtained numerical results are listed in Table II. To avoid numerical difficulty, we take the low limit of integration of $S_{G}$ in Eq. (31) to be $10^{-7}$. Due to the

TABLE II. Theoretical GSR values, using the MPT model, at various $Q^{2}$.

\begin{tabular}{lc}
\hline \hline $\mathbf{Q}^{2} \mathrm{GeV}^{2}$ & $\mathbf{S}_{\mathbf{G}}$ \\
\hline 0.15 & 0.325 \\
0.21 & 0.312 \\
0.27 & 0.301 \\
0.313 & 0.294 \\
4 & 0.196 \\
\hline
\end{tabular}

nonexistence of gluon radiation at low energy scales, the probability of the appearance of sea quarks is very low and it is expected that the $S_{G}$ value approaches $\frac{1}{3}$. The $S_{G}$ values at low energy scales listed in Table II confirm this reality.

\section{SUMMARY AND CONCLUSION}

Considering the nonexistence of the pQCD coupling at low spacelike momenta $0<Q^{2} \lesssim \Lambda$, we employed an approach called anQCD for the purpose of reforming and modifying the calculations at an energy scale $Q^{2}<\Lambda^{2}$ to evaluate the unpolarized nucleon structure function at the mentioned momenta. In this way, considering the importance of gluon density in the singlet sector of nucleon structure function computations, we applied the anQCD approach based on the MPT model, where a mass is attributed to gluon. Using this approach, specifically the MPT model, the NSF is calculable at all energy scales $Q^{2}>0$ where at moderate and high energies the MPT results for the NSF match those of the underlying pQCD. It was seen that at low energies the behavior of $F_{2}\left(x, Q^{2}\right)$ is smoother than in the underlying pQCD. We showed these facts in Fig. 2 at $Q^{2}=0.15,0.21,0.27$, and $0.313 \mathrm{GeV}^{2}$. Consequently, with due attention to the acceptable conformity between MPT results and available data, we conclude that the results of the anQCD approach using the MPT model are more reliable than those of the (underlying) pQCD at low energies.

Also, we evaluated the Gottfried sum rule while considering a nucleon sea flavor asymmetry $\left[\bar{u}\left(x, Q^{2}\right)<\right.$ $\left.\bar{d}\left(x, Q^{2}\right)\right]$. The naive GSR indicates a difference in the value with respect to the experimental data, because according to the naive parton model for the GSR $S_{G}=1 / 3$, but experimental data shows a deviation from $\frac{1}{3}$. By applying the anQCD approach (specifically the MPT model), we achieved a result closer to the experimental data. In addition to the experimental energy scale $Q^{2}=4 \mathrm{GeV}^{2}$, we employed this model at energy scales $Q^{2}=0.15,0.21,0.27$, and $0.313 \mathrm{GeV}^{2}$ due to the applicability of this approach at low energies. Numerical results for $S_{G}$ at low energy scales, based on the MPT model, are in agreement with the behavior of the parton densities, which is the correct behavior at these scales.

The anQCD approach can be employed to calculate nuclear structure functions like ${ }^{3} \mathrm{He}$ and ${ }^{7} \mathrm{Li}$ where there are many experimental data for them at low energy scales. We hope to report on this subject in future.

\section{ACKNOWLEDGMENTS}

S. A. T. is grateful to the School of Particles and Accelerators, Institute for Research in Fundamental Sciences (IPM) to make the required facilities to do this project. The rest of authors are thankful Yazd university to provide the warm hospitality in connection to this research project. 
[1] S. J. Brodsky, G. F. de Teramond, and A. Deur, Nonperturbative QCD coupling and its $\beta$-function from light-front holography, Phys. Rev. D 81, 096010 (2010).

[2] Y. L. Dokshitzer, G. Marchesini, and B. R. Webber, Dispersive approach to power behaved contributions in QCD hard processes, Nucl. Phys. B469, 93 (1996).

[3] Y. L. Dokshitzer and B. R. Webber, Calculation of power corrections to hadronic event shapes, Phys. Lett. B 352, 451 (1995).

[4] D. V. Shirkov and I. L. Solovtsov, Analytic Model for the QCD Running Coupling with Universal $\alpha_{s}(0)$ Value, Phys. Rev. Lett. 79, 1209 (1997).

[5] K. A. Milton and I. L. Solovtsov, Analytic perturbation theory in QCD and Schwinger's connection between the beta function and the spectral density, Phys. Rev. D 55, 5295 (1997).

[6] K. A. Milton, I. L. Solovtsov, and O. P. Solovtsova, Analytic perturbation theory and inclusive tau decay, Phys. Lett. B 415, 104 (1997).

[7] K. A. Milton and O.P. Solovtsova, Analytic perturbation theory: A new approach to the analytic continuation of the strong coupling constant $\alpha_{s}$ into the timelike region, Phys. Rev. D 57, 5402 (1998).

[8] D. V. Shirkov, Renorm-group, causality and nonpower perturbation expansion in QFT, Theor. Math. Phys. 119, 438 (1999).

[9] D. V. Shirkov, Analytic perturbation theory for QCD observables, Theor. Math. Phys. 127, 409 (2001).

[10] L. Ghasemzadeh, A. Mirjalili, and S. Atashbar Tehrani, Nonsinglet polarized nucleon structure function in infraredsafe QCD, Phys. Rev. D 100, 114017 (2019).

[11] A. P. Bakulev, S. V. Mikhailov, and N. G. Stefanis, QCD analytic perturbation theory: From integer powers to any power of the running coupling, Phys. Rev. D 72, 074014 (2005).

[12] A. P. Bakulev, S. V. Mikhailov, and N. G. Stefanis, Fractional analytic perturbation theory in Minkowski space and application to Higgs boson decay into a b anti-b pair, Phys. Rev. D 75, 056005 (2007); Erratum, Phys. Rev. D 77, 079901 (2008).

[13] A. P. Bakulev, S. V. Mikhailov, and N. G. Stefanis, Higherorder QCD perturbation theory in different schemes: From FOPT to CIPT to FAPT, J. High Energy Phys. 06 (2010) 085 .

[14] C. Ayala and G. Cvetič, anQCD: A Mathematica package for calculations in general analytic QCD models, Comput. Phys. Commun. 190, 182 (2015).

[15] C. Ayala, C. Contreras, and G. Cvetič, Extended analytic QCD model with perturbative QCD behavior at high momenta, Phys. Rev. D 85, 114043 (2012).

[16] C. Ayala, G. Cvetič, R. Kögerler, and I. Kondrashuk, Nearly perturbative lattice-motivated QCD coupling with zero IR limit, J. Phys. G 45, 035001 (2018).

[17] D. V. Shirkov, 'Massive' Perturbative QCD, regular in the IR limit, Phys. Part. Nucl. Lett. 10, 186 (2013).
[18] C. Ayala and S. V. Mikhailov, How to perform a QCD analysis of DIS in analytic perturbation theory, Phys. Rev. D 92, 014028 (2015).

[19] G. Cvetic and A. V. Kotikov, Analogs of noninteger powers in general analytic QCD, J. Phys. G 39, 065005 (2012).

[20] E. G. Floratos, C. Kounnas, and R. Lacaze, Higher order QCD effects in inclusive annihilation and deep inelastic scattering, Nucl. Phys. B192, 417 (1981).

[21] A. Vogt, Efficient evolution of unpolarized and polarized parton distributions with QCD-PEGASUS, Comput. Phys. Commun. 170, 65 (2005).

[22] A. Vogt, S. Moch, and J. A. M. Vermaseren, The Three-loop splitting functions in QCD: The singlet case, Nucl. Phys. B691, 129 (2004).

[23] J. Koekoek and R. Koekoek, Differential equations for generalized Jacobi polynomials, J. Comput. Appl. Math. 126, 1 (2000).

[24] A. L. Kataev, A. V. Kotikov, G. Parente, and A. V. Sidorov, Next to next-to-leading order QCD analysis of the CCFR data for $\mathrm{xF} 3$ and $\mathrm{F} 2$ structure functions of the deep inelastic neutrino-nucleon scattering, Phys. Let. B388, 179 (1996).

[25] A. L. Kataev, A. V. Kotikov, G. Parente, and A. V. Sidorov, Next to next-to-leading order QCD analysis of the revised CCFR data for $\mathrm{xF} 3$ structure function and the higher twist contributions, Phys. Lett. B 417, 374 (1998).

[26] P. Jimenez-Delgado and E. Reya, Delineating parton distributions and the strong coupling, Phys. Rev. D 89, 074049 (2014).

[27] M. R. Adams et al. (E665 Collaboration), Proton and deuteron structure functions in muon scattering at 470GeV, Phys. Rev. D 54, 3006 (1996).

[28] A. Cafarella, C. Coriano, and M. Guzzi, Nnlo logarithmic expansions and exact solutions of the DGLAP equations from X-space: New algorithms for precision studies at the lhc, Nucl. Phys. B748, 253 (2006).

[29] W. L. van Neerven and A. Vogt, NNLO evolution of deep inelastic structure functions: The nonsinglet case, Nucl. Phys. B568, 263 (2000).

[30] W. L. van Neerven and A. Vogt, NNLO evolution of deep inelastic structure functions: The singlet case, Nucl. Phys. B588, 345 (2000).

[31] P. Jimenez Delgado, Dynamical parton distributions of the nucleon up to NNLO of QCD, arXiv:0902.3947.

[32] P. Amaudruz et al., The Gottfried Sum from the Ratio F2(n)/ F2(p), Phys. Rev. Lett. 66, 2712 (1991).

[33] D. J. Broadhurst, A. L. Kataev, and C. J. Maxwell, Comparison of the Gottfried and Adler sum rules within the large-Nc expansion, Phys. Lett. B 590, 76 (2004).

[34] M. Arneodo et al., A reevaluation of the Gottfried sum, Phys. Rev. D 50, R1 (1994).

[35] E.-M. Kabuss, Final results from the NMC, AIP Conf. Proc. 407, 291 (1997).

[36] R. Abbate and S. Forte, Re-evaluation of the Gottfried sum using neural networks, Phys. Rev. D 72, 117503 (2005). 